\title{
Radiation recall dermatitis due to gemcitabine does not suggest the need to discontinue chemotherapy
}

\author{
MICHAEL LOCK ${ }^{1}$, KEVIN SINCLAIR ${ }^{1}$, STEPHEN WELCH $^{2}$, \\ JAWAID YOUNUS $^{2}$ and MOHAMMAD SALIM ${ }^{1}$ \\ Divisions of ${ }^{1}$ Radiation Oncology, and ${ }^{2}$ Medical Oncology, \\ London Regional Cancer Program, London, Ontario N6A 4L6, Canada
}

Received June 17, 2010; Accepted August 26, 2010

DOI: $10.3892 / \mathrm{ol} .2010 .195$

\begin{abstract}
Radiation recall is common following treatment with certain chemotherapy drugs and presents frequently as a skin reaction. With gemcitabine, such a recall phenomenon may affect internal tissues and presents itself as myositis. Although such reactions have previously been reported in the literature, whether or not to continue chemotherapy during such reactions remains controversial. We reported a case of radiation recall in a patient treated with gemcitabine and radiation therapy that presented as myositis. We were able to continue palliative chemotherapy and manage the side effects with supportive care treatment. This case report provides partial support for the continuation of chemotherapy when required even when a recall reaction is encountered.
\end{abstract}

\section{Introduction}

Radiation recall is described as inflammation occurring in previously irradiated areas which is triggered by the administration of a drug (1). It is most commonly observed when chemotherapeutic drugs, such as anthracyclines, taxanes, alkylating agents, 5-fluorouracil and capecitabine, are administered shortly after radiation, although the reaction may occur years after the completion of radiation $(1,2)$. Radiation recall most often manifests as inflammatory reactions of the skin but may also occur in internal organs and tissues $(1,2)$. In the event of such a reaction, the offending drug is discontinued. This case report evaluates a patient with poorly differentiated adenocarcinoma of the liver. The patient had a recall reaction in the form of myositis as a result of treatment with gemcitabine. Radiation recall induced by gemcitabine is a rare and relatively new phenomenon in the literature. Only two other cases of radiation recall in a patient being treated for cancer of the liver have been reported $(3,4)$, and no cases exist involving primary cancer. It

Correspondence to: Dr Michael Lock, London Regional Cancer Program, 790 Commissioners Rd. E., London, Ontario N6A 4L6, Canada

E-mail:michael.lock@lhsc.on.ca

Key words: radiation recall, gemcitabine, myositis has been reported that the majority of cases of radiation recall induced by gemcitabine administration involve inflammation of internal tissues and organs, which differs from the majority of reactions caused by other chemotherapeutic agents, as stated above (2). In this case report, continuing gemcitabine treatment during radiation recall was analyzed and the related current literature was evaluated.

\section{Case report}

A 50-year-old woman initially presented with right flank discomfort in January 2008. Multiple lesions were evident in the liver, and a needle biopsy confirmed the presence of poorly differentiated adenocarcinoma. Due to the fact that multiple lesions were located in multiple lobes, the patient was not a candidate for surgery and was therefore considered for radiation therapy followed by chemotherapy with palliative intent. The patient received radiation therapy for a total dose of $44.1 \mathrm{~Gy}$ in 15 fractions, with a biological equivalent dose of 58.5 Gy. The treatment was well tolerated, with no side effects greater than the National Cancer Institute of Canada Grade I to the radiation.

The patient was started on gemcitabine 8 weeks after completion of radiation. She received a dose of $1000 \mathrm{mg} / \mathrm{m}^{2}$ on days 1 and 8 of a 3 -week cycle. On day 8 of her fourth cycle, the patient complained of a discomfort in the previously irradiated field. An abdominal examination revealed a welldemarcated $15-\mathrm{cm}$ rectangular indurated area. The overlying skin was erythematous and slightly tender to palpation. Consistent with the literature, clinical and radiological images (Figs. 1 and 2), it was determined that she presented with a radiation recall reaction induced by gemcitabine treatment, in the form of myositis.

Following consultation with the patient, the decision was made to continue with the gemcitabine treatment as the symptoms appeared to be improving in response to this treatment. The patient was then started on ibuprofen 200 to 400 mg three times a day for 6 weeks, vitamin E 400 IU two times a day and vitamin C $500 \mathrm{mg}$ three times a day. She continued with two more cycles of chemotherapy and had a documented stable disease response. Subsequently, during a follow-up examination, the patient reported that the discomfort caused by the recall reaction had continued to subside. On visual 


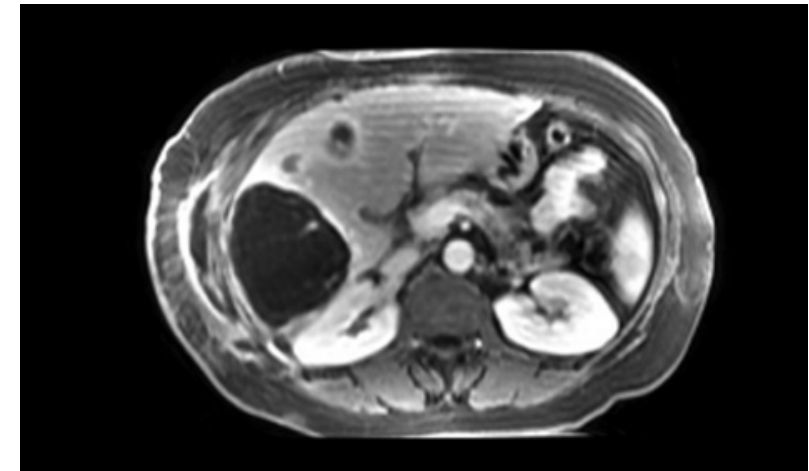

Figure 1. MRI image shows a dominant mass in the right hepatic lobe posterior segment measuring approximately $12 \times 8 \mathrm{~cm}$ with rim enhancement. A decrease in signal intensity post contrast with mainly rim type enhancement is noted. The induration observed along the right lateral margin of the lower chest wall and abdomen of the patient is diffuse with the rim enhancement of the muscle.

examination, the reaction appeared to have decreased in size as well.

\section{Discussion}

Radiation recall occurs when an inciting agent, such as a chemotherapeutic drug, is administered after radiation. These agents most commonly produce reactions such as dermatitis or myositis, but can also produce rarely observed reactions such as optic neuritis, brainstem necrosis and erysipeloid lesions $(1,5)$. The first reported case of radiation recall was in 1959 and was attributed to actinomycin-D (6). Although the term radiation recall and its implications are well known and various other agents have been found to cause an occurrence, less than 150 cases have been published in the literature. The majority of these cases have been reported since the turn of the century and are likely associated with the discovery and increased use of new cytotoxic agents. The exact cause or mechanism remains unknown, which is complicated by the fact that a variety of drugs have been found to induce radiation recall with different chemical, biological and metabolic characteristics. In addition, the timing of the occurrence of radiation recall has remained variable, and no particular risk factors from the patient angle have been defined.

Gemcitabine is an anti-metabolite nucleoside analog that is used against tumors such as pancreatic and lung carcinoma. Recall reactions attributed to gemcitabine are infrequently reported in the literature. A literature search of Pub Med, revealed only 28 cases since the first report in 1999 (1-5,7-21). Hird et al reported that gemcitabine was involved only 9 times out of 75 cases of radiation recall dermatitis since 1959 (7).

A review of the literature provides some practical insights into this phenomenon. Table I summarizes our case along with all other published radiation recall reactions related to gemcitabine. In 2004, Friedlander et al described that the majority of cases of radiation recall related to gemcitabine involved internal tissues and organs (2). However, our study showed that $50 \%$ of such cases involved only skin and another $18 \%$ of cases involved both skin and internal tissues. Due to the paucity of data, we were not able to correlate radiation

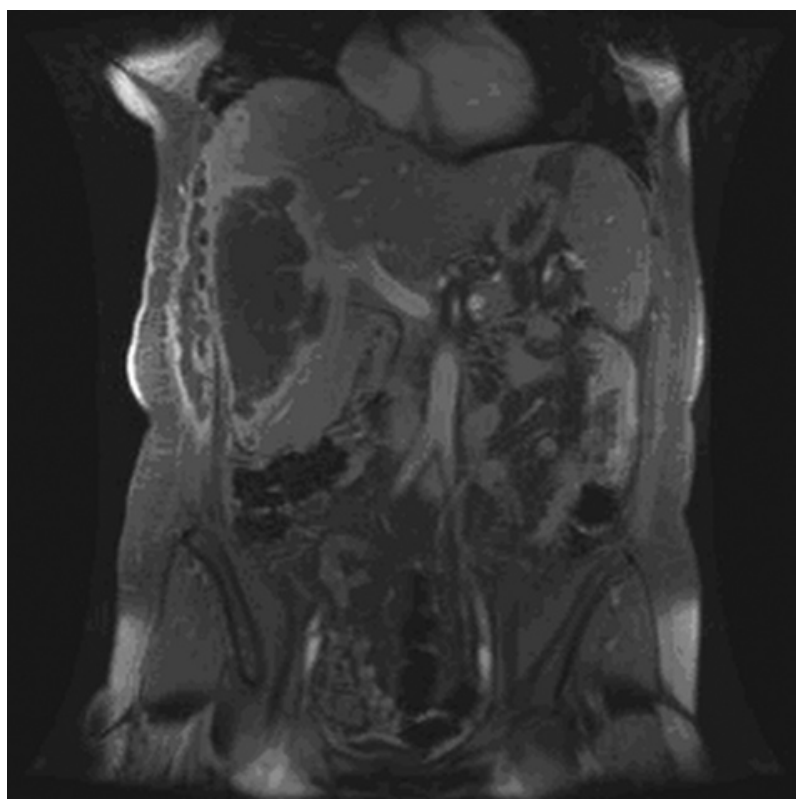

Figure 2. Coronal MRI showing the relative location of the liver lesion and muscle thickening.

dose or dose per fraction with severity or frequency. In most of the cases, radiation dose to the skin is likely to have been lower than the dose to the internal structures. Therefore, it can be suggested that the radiation dose does not appear to affect the risk of radiation recall.

In that same study, Friedlander et al also documented that a shorter time interval between radiation therapy and chemotherapy was correlated with recall reactions involving internal tissues (2). The averages of this interval confirm this in that the average time period for cutaneous reactions was 4 months while the average time period for reactions involving internal tissues was 2.5 months, although the medians were found to be the same at 1.5 months. The relationship between the interval from commencement of chemotherapy and the type of reaction suggests a variable sensitivity.

Another significant observation, noted by Camidge and Price in 2001, is that the risk of succumbing to a recall reaction is not affected by whether the patient receives monotherapy or if chemotherapeutic agents are administered in combination (22). We also noted that there is no significant difference between the number of gemcitabine-induced radiation recall reactions presented while the patient is receiving monotherapy or a combination treatment, nor does this appear to affect the type of reaction presented. In our case, gemcitabine administration was continued while the recall reaction was treated with conservative supportive care. Only six other studies in the literature of radiation recall induced by gemcitabine report the continuation of gemcitabine treatment, whether to maintain the current regimen or lower the dose $(1,9,10,12,15)$. Four of these cases reported that the patients had complete improvement of their symptoms while still on gemcitabine. The other two cases reported that the symptoms improved, but then recurred following each administration. It should be noted that in one of these cases the patient received no treatment for the reaction (12). Including our case, none of the cases in which gemcitabine was continued noted an 


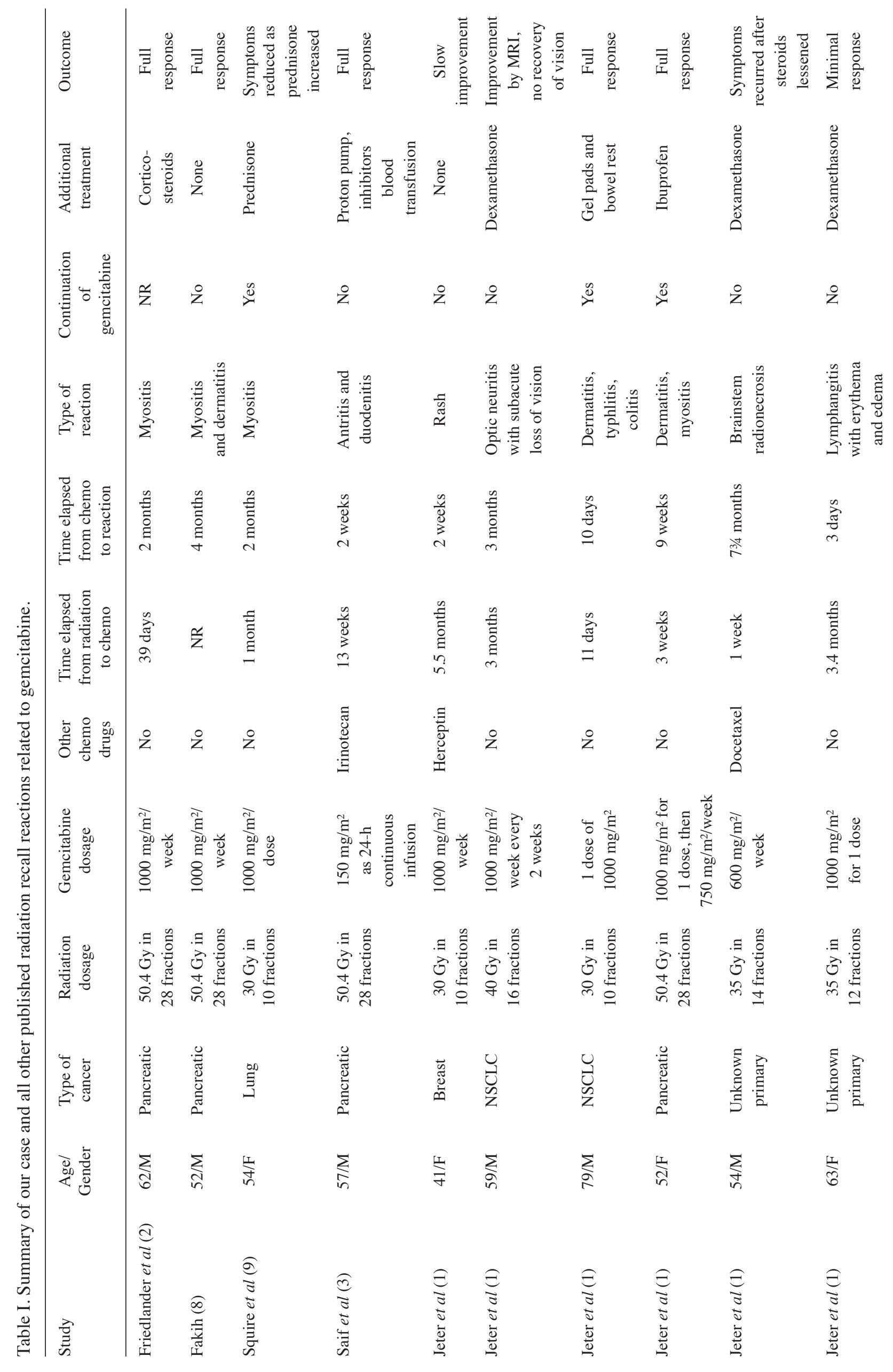




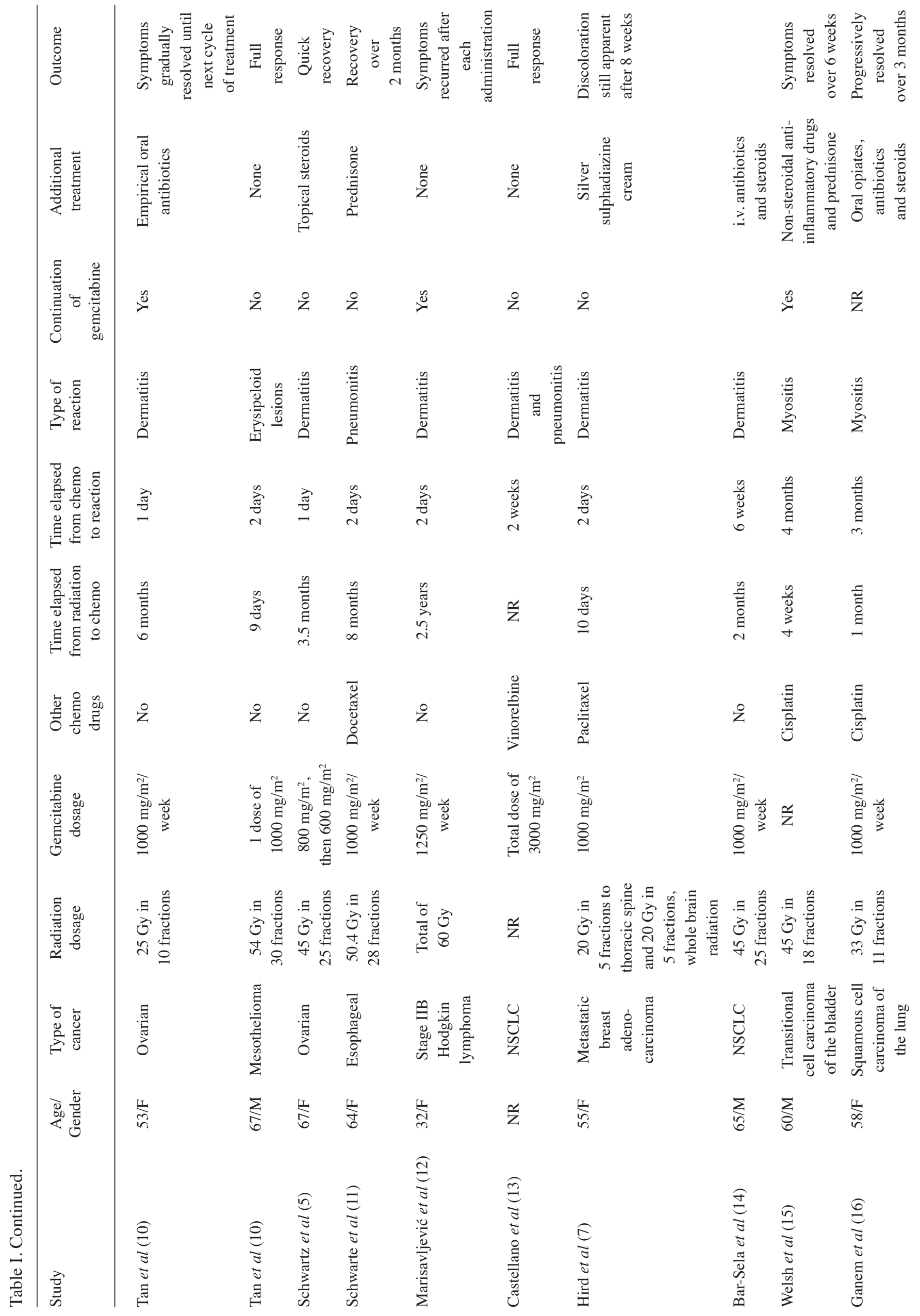




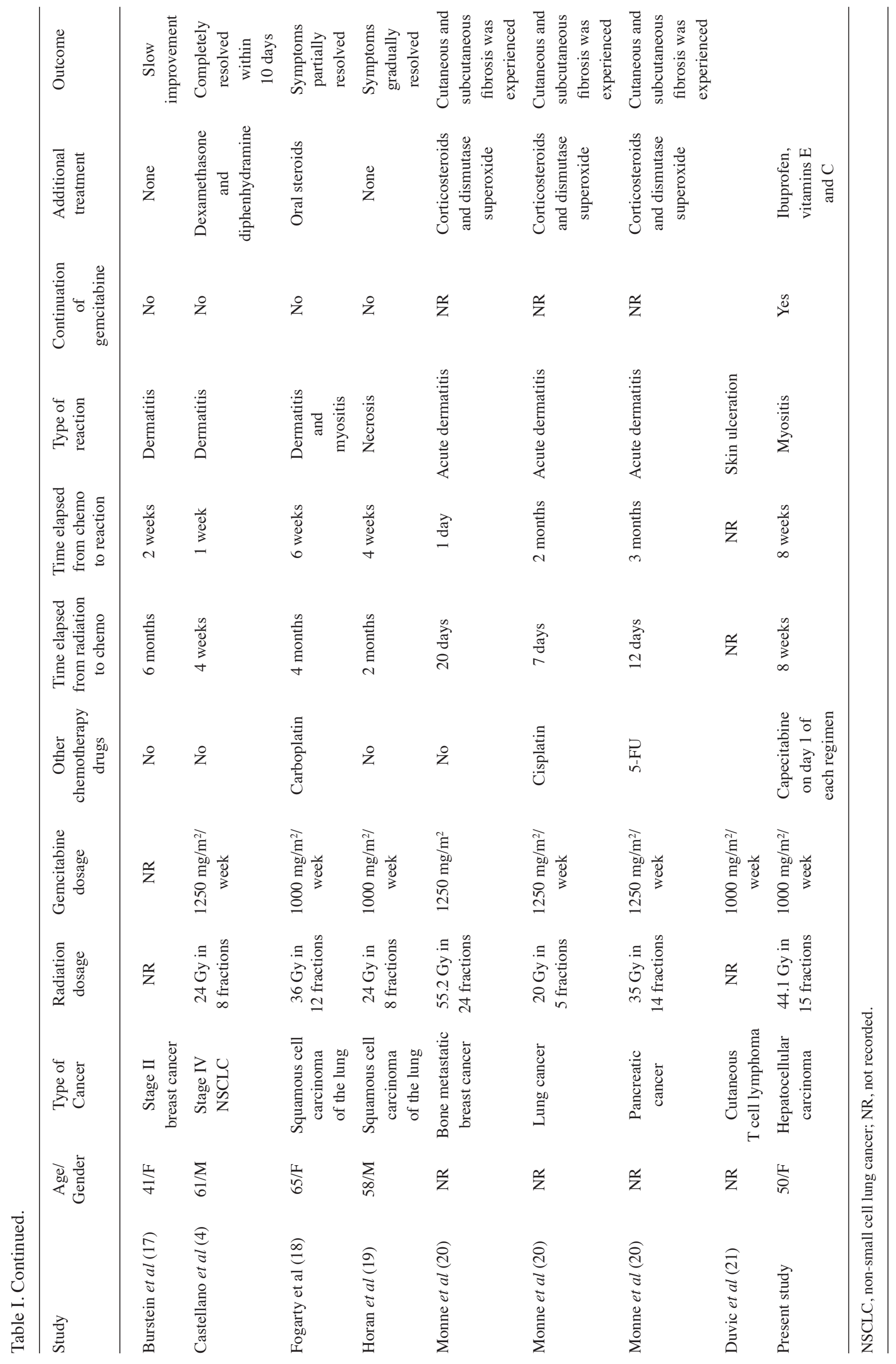


increase in symptoms or pain at any time during chemotherapy. In the case in which the symptoms recurred after each administration, the symptoms returned to their original form and did not present at a higher grade $(10,12)$. Moreover, two of the cases in the literature documenting a discontinuation of gemcitabine treatment reported that the cancer had metastasized, leading to patient death $(8,19)$. Clearly this is a primary consideration for the patient and health care provider when a reaction occurs. In our case, the symptoms experienced by the patient gradually improved while on gemcitabine treatment and did not worsen after administration.

Our case report, along with other similar cases in the literature, lends support to treating clinicians who decide to continue chemotherapy with gemcitabine in cases with a radiation recall reaction. Our data do not suggest that a gemcitabine recall reaction heralds a more resistant disease or greater metastatic potential. Radiation techniques or regimens do not need to be adjusted. Gemcitabine recall remains an enigmatic and rare event that should not affect primary cancer management decisions. Patients can be informed that the reaction usually resolves and does not change their prognosis.

\section{References}

1. Jeter M, Jänne $\mathrm{P}$, Brooks $\mathrm{S}$, et al: Gemcitabine-induced radiation recall. Int J Radiat Oncol Biol Phys 53: 394-400, 2002.

2. Friedlander P, Bansal R, Schwartz L, Wagman R, Posner J and Kemeny N: Gemcitabine-induced radiation recall preferentially involves internal tissues and organs. Cancer 100: 1793-1799, 2004.

3. Saif M, Sellers S and Russo S: Gemcitabine-related radiation recall in a patient with pancreatic cancer. Anticancer Drugs 17: 107-111, 2006.

4. Castellano D, Hitt R, Cortés-Funes H, Romero A and RodriguesPeralto J: Diagnosis in oncology. Case 2. Radiation recall reaction induced by gemcitabine. J Clin Oncol 3: 695-696, 2000.

5. Schwartz B, Khuntia D, Kennedy A and Markham M: Gemcitabine-induced radiation recall dermatitis following whole pelvic radiation therapy. Gynecol Oncol 91: 421-422, 2003.

6. D'Angio G, Farber S and Maddock C: Potentiation of X-ray effects of actinomycin D. Radiology 73: 174-177, 1959.
7. Hird A, Wilson J, Symons S, Sinclair E, Davis M and Chow E: Radiation recall dermatitis: case report and review of the literature. Curr Oncol 15: 53-62, 2008.

8. Fakih M: Gemcitabine-induced rectus abdominus radiation recall. JOP 7: 306-310, 2006.

9. Squire S, Chan M, Feller E, Mega A and Gold R: An unusual case of gemcitabine-induced radiation recall. Am J Clin Oncol 29: 636, 2006.

10. Tan D, Bunce P, Liles W and Gold W: Gemcitabine-related 'pseudocellulitis': report of 2 cases and review of the literature. Clin Infect Dis 45: e72-e76, 2007.

11. Schwarte S, Wagner K, Karstens J and Bremer M: Radiation recall pneumonitis induced by gemcitabine. Strahlenther Onkol 183: 215-217, 2007

12. Marisavljević D, Ristić B and Hajder J: Gemcitabine-induced radiation recall dermatitis in a patient with resistant Hodgkin lymphoma. Am J Hematol 80: 91, 2005.

13. Castellano D, Hitt R, Ciruelos E, Cortés-Funes H and Colomer R Biweekly vinorelbine and gemcitabine: a phase I dose-finding study in patients with advanced solid tumors. Ann Oncol 14: 783-787, 2003.

14. Bar-Sela G, Beny A, Bergman R and Kuten A: Gemcitabineinduced radiation recall dermatitis: case report. Tumori 87: 428-430, 2001.

15. Welsh J, Torre T, DeWeese T and O'Reilly S : Radiation myositis. Ann Oncol 10: 1105-1108, 1999.

16. Ganem G, Solal-Celigny P, Joffroy A, Tassy D, Delpon A and Dupuis O: Radiation myositis: The possible role of gemcitabine. Ann Oncol 11: 1615-1616, 2000.

17. Burstein H: Diagnosis in oncology. Case 1. Radiation recall dermatitis from gemcitabine. Am J Clin Oncol 18: 693-698, 2000.

18. Fogarty G, Ball D and Rischin D: Radiation recall reaction following gemcitabine. Lung Cancer 33: 299-302, 2001.

19. Horan G, Smith S and Podd T: Gemcitabine-induced radiation necrosis of the pectoralis major muscle. Clin Oncol 18: 85-91, 2006.

20. Sole Monne JM, Macia Garau M, Cambra Serest MJ and Sureda Gonzalez M: Radiation recall dermatitis from gemcitabine. a new toxic effect in 3 patients. Radiother Oncol 57: S32-S34, 2000

21. Duvic M, Talpur R, Wen S, Kurzrock R, David CL and Apisarnthanarax N: Phase II evaluation of gemcitabine monotherapy for cutaneous T-cell lymphoma. Clin Lymphoma Myeloma 7: 51-58, 2006.

22. Camidge $\mathrm{R}$ and Price A: Characterizing the phenomenon of radiation recall dermatitis. Radiother Oncol 59: 237-245, 2001. 